\title{
Cancer of Unknown Primary: What Kind of Chemotherapy? What Kind of Disease?
}

\author{
George Pentheroudakis Nicholas Pavlidis \\ Department of Medical Oncology, loannina University Hospital, Greece
}

Cancer of unknown primary (CUP) ranks as the fourth to seventh cause of cancer death in developed societies and is a diagnosis of exclusion: a standardised diagnostic work-up that includes physical examination, imaging and pathology is necessary in order to exclude identification of a primary tumour. As with any other entity that is defined by absence of a characteristic (the primary site, in this case), it used to be an extremely heterogeneous disease. Different tumours with distinct molecular signatures and biology contribute to this grouping. A few years ago, the definition of CUP subsets with favourable prognosis that are characterised by biology and behaviour that is similar to known metastatic primary tumours eliminated some of the inherent heterogeneity of CUP [1]. Patients with serous peritoneal adenocarcinomatosis, isolated axillary lymph nodes involved by adenocarcinoma, PSA-expressing bony metastases or squamous upper/ mid-cervical lymphadenopathy probably harbour occult tumours in the peritoneum, breast, prostate or head/neck, respectively and fare as well as patients with the relevant metastatic carcinomas. Patients harbouring adenocarcinoma or poorly differentiated carcinoma of unknown primary with multiple visceral metastases, often in peculiar sites, no primary-related signs or symptoms, resistance to therapy and dismal prognosis make up the 'poor risk' CUP category that is regarded by many investigators as the 'genuine' CUP entity [2].

In this issue, Schuette et al. present a single-arm phase II study evaluating the efficacy and safety of the capecitabine/ oxaliplatin combination (CapOx) in patients with poor-risk CUP [3]. The evaluated combination of a convenient oral fluoropyrimidine coupled to a new-generation platinum compound has proven activity in tumours of the gastrointestinal tract and promising activity against breast, ovarian, pancreatic, lung tumours. Since autopsy and microarrray series showed that the occult CUP primary resides in the lung, pancreas, lower GI tract or breast in three quarters of cases, the choice seems to be rational indeed [4]. The study had $80 \%$ power to detect an improvement of its primary endpoint, response rate, from $20 \%$ to $>35 \%$ with a type I error of $5 \%$. The convenience and safety of CapOx was satisfactory, with $37 \%$ of the 51 patients enrolled experiencing any grade 3 or 4 sideeffect and 1 toxic death. The most common severe toxicities were fatigue, diarrhoea and anaemia, each occurring in less than $10 \%$ of patients. Grade 3 neurotoxicity, a dose-limiting effect of oxaliplatin, occurred in $2 \%$. Still, a disappointing tumour regression rate of only $11.7 \%$ was reported, with equally disappointing median progression-free and overall survival times of 2.5 and 7.5 months in patients who were fit (PS 0-1) in $80 \%$ of cases. The small sample size of the study precludes any subgroup analyses for reliable identification of prognostic factors. As the authors state, the $95 \%$ confidence intervals of 4-24\% for the response rate make it extremely unlikely for any future study to demonstrate a superiority of CapOx over standard taxane/platinum-based schedules. The latter resulted in remission rates of $20-40 \%$ in published trials, still failing themselves to prolong median survival of poor-risk CUP patients beyond the 1-year benchmark.

This recent failure to improve the outcome of patients with poor-risk CUP confirms results from previous studies. We recently reviewed 20 clinical trials that enrolled a total of 1,319 poor-risk CUP patients to various chemotherapy regimens and showed that responses invariably occurred in a minority of patients $(20-45 \%)$ while the median survival did not surpass 1 year [5]. Moreover, we established that the outcome of CUP patients treated with platinum, taxanes or GI-type regimens was clearly inferior to that of patients with metastatic breast or colon carcinomas, despite the fact that microarrays identify a breast or colon primary for $>50 \%$ of CUP cases. This leaves us with a key question of what is missing in CUP: is it the primary site or the biology?

\section{KARGER}

Fax +497614520714

Information@Karger.de

www.karger.com
(C) 2009 S. Karger GmbH, Freiburg

Accessible online at:

www.karger.com/onk
George Pentheroudakis, MD

Department of Medical Oncology

Ioannina University Hospital

Niarxou Avenue, 45500 Ioannina, Greece

Tel. and Fax +30 2651099394

gpenther@otenet.gr 
High-throughput technologies that profile expression of tens or hundreds of genes or proteins recently emerged. These platforms have been able to biologically identify the primary tumour in blinded experiments with $70-85 \%$ accuracy. They have also been used in order to classify CUP cases to a primary tissue of origin according to the observed multigene expression profile of each CUP which was compared to a standard gene expression profile of each known solid tumour. The rationale for this strategy of biological identification of a primary for each CUP case is to allow administration of primary-specific, effective therapy, i.e. bevacizumab for a 'colon CUP' [6]. Recently Varadhachary et al. suggested that a CK20+ and/or CDX2+, CK7- immunohistochemical profile identifies CUP with an occult colon primary, a finding that could be worth validating among responders in the current trial by Schuette. However, the assumption that a 'colon CUP' behaves as a typical metastatic colon carcinoma has not been proven yet. Some investigators postulate that CUP tumours have not only a 'primary tissue-specific' but also a 'pro-metastatic and primary regressive' molecular signature that differentiates their biological behaviour from that of metastatic solid tumours of known primary [2]. If this is the case, a 'colon
CUP' would behave and respond to therapy differently from a typical metastatic colon cancer of known primary. Other investigators propose that assignment of a 'primary' to each CUP does not take advantage of the dynamic of profiling molecular platforms; instead, this powerful technology should be exploited in order to identify the molecular aberrations of each individual CUP tumour on which tumour survival depends, target them with smart drugs and eradicate the malignant clone [7].

Ultimately, the improvement of the outcome of patients with poor-risk CUP by means of biological classification to a primary site and administration of primary-specific therapy should be established in prospective clinical trials. Such a trial would randomize poor-risk CUP patients to empiric taxane/ platinum therapy or to molecular profiling and primary-specific therapy. Until such a trial takes place, high-throughput molecular profiling platforms could be better suited to unravelling the molecular aberrations characterising CUP and to identifying biomolecular targets for modulation. It still remains to be seen whether the proper therapeutic approach for CUP patients will be primary-specific, CUP-specific or patient-specific therapy.

\section{References}

1 Pavlidis N, Briasoulis E, Hainsworth J, Greco FA: Diagnostic and therapeutic management of cancer of unknown primary. Eur J Cancer 2003;39:19902005.

2 Pentheroudakis G, Briasoulis E, Pavlidis N: Cancer of unknown primary site: missing primary or missing biology? Oncologist 2007;12:418-425.

3 Schuette K, Folprecht G, Kretzschmar A, Link H, Kohne CH, Grunwald V, Stahl M, Huebner G: Phase II trial of capecitabine and oxaliplatin in patients with adeno- and undifferentiated carcinoma of unknown primary. Onkologie 2009;32: DOI 10.1159/000201125.
4 Pentheroudakis G, Golfinopoulos V, Pavlidis N: Switching benchmarks in cancer of unknown primary: from autopsy to microarray. Eur J Cancer 2007:43:2026-2036.

5 Pentheroudakis G, Greco FA, Pavlidis N. Molecular assignment of tissue of origin in cancer of unknown primary may not predict response to therapy or outcome: A systematic literature review. Cancer Treat Rev 2008; DOI 10.1016/j.ctrv.2008.10.003.
6 Varadhachary GR, Talantov D, Raber MN, Meng C, Hess KR, Jatkoe T, Lenzi R, Spigel DR, Wang Y, Greco FA, Abbruzzese JL, Hainsworth JD: Molecular profiling of cancer of unknown primary and correlation with clinical evaluation. J Clin Oncol 2008;26:4442-4448.

7 Wulfkuhle JD, Speer R, Pierobon M, Laird J, Espina V, Deng J, Mammano E, Yang SX, Swain SM, Nitti D, Esserman LJ, Belluco C, Liotta L, Petricoin EF: Multiplexed cell signaling analysis of human breast cancer: applications for personalized therapy. J Proteome Res 2008;7:1508-1517. 